\title{
Endoscopic internal drainage as first-line treatment for fistula following gastrointestinal surgery: a case series
}

Authors

Institutions
Gianfranco Donatelli ${ }^{1}$, Jean-Loup Dumont ${ }^{1}$, Fabrizio Cereatti ${ }^{2}$, Parag Dhumane ${ }^{3}$, Thierry Tuszynski ${ }^{1}$, Bertrand Marie Vergeau', Bruno Meduri ${ }^{1}$

${ }^{1}$ Unité d’Endoscopie Interventionnelle, Ramsay Générale de Santé, Hôpital Privé des Peupliers, Paris, France

${ }^{2}$ Unit of Interventional Endoscopy, Department of General Surgery “P. Stefanini”, "Sapienza” University of Rome, Rome, Italy

${ }^{3}$ Department of General and Laparoscopic Surgery, Lilavati Hospital and Research Center, Bandra(w), Mumbai, India submitted

16. December 2015 accepted after revision 21. February 2016

\section{Bibliography}

DOI http://dx.doi.org/

10.1055/s-0042-105206

Published online: 15.4.2016

Endoscopy International Open

2016; 04: E647-E651

(c) Georg Thieme Verlag KG

Stuttgart · New York

E-ISSN 2196-9736

\section{Corresponding author}

Gianfranco Donatelli

Unité d'Endoscopie

Interventionnelle

Ramsay Générale de Santé,

Hôpital Privé des Peupliers

8 Place de l'Abbé G. Hénocque

75013, Paris

France

Fax: +33-01-44-16-56-15

donatelligianfranco@gmail.com
Background and study aims: Leaks following gastrointestinal surgery are a dreadful complication burdened by high morbidity and not irrelevant mortality.

\section{Introduction \\ $\nabla$}

Endoscopic internal drainage (EID) has been recently introduced for treatment of leaks and fistulas following bariatric surgery. EID may be applied both as first-line treatment and as rescue therapy [1,2]. Fistulas following gastrointestinal surgery both for benign and malign pathologies remain a major clinical problem. Such complications account for significant morbidity and a mortality rate that can reach $60 \%$ if treatment is delayed [3]. Here we report our experience with EID as first-line treatment of fistula following different types of gastrointestinal surgery. To our knowledge, this is the first case series in which EID has been used systematically for fistulas following both upper and lower gastrointestinal surgery.

\section{Patients and methods \\ $\nabla$}

From March 2013 to November 2015, 11 patients were treated at our endoscopic tertiary center for fistula following gastrointestinal surgery. The conditions with which these patients were diagnosed and procedures that they had undergone are as follow: four duodenal fistulas (biliopancreatic surgery), two colic fistulas (colorectal surgery), and five esophagogastric-jejunal fistulas (foregut surgery). Data were collected in a prospectively maintained database and were retrospectively analyzed. All patients were referred to our endoscopy department by the surgical team performing the surgical procedure. Informed consent including the necessity of multiple endo-
Endoscopic internal drainage (EID) has showed optimal results in the treatment of leaks following bariatric surgery. We report our experience with EID as first-line treatment for fistulas following surgery along all gastrointestinal tract.

scopic sessions was obtained from all patients. The study was approved by Institutional Review Board for Human Research.

Patient features and demographics are listed in - Table1. Delay between first surgery and EID averaged 14 days (range 3-60). Ten of 11 patients had previously positioned surgical or radiological drainage. All patients presented either clinical evidence of sepsis (leukocytosis, fever, elevation on polymerase chain reaction) or output of enteric fluid from the drainage or medium contrast extravasation at swallow study/pelvic computed tomography scans. Opacification of fistula was always performed during endoscopic examination in order to evaluate the fistula opening, the collection, its shape and size. Endoscopic evaluation was of paramount importance to correctly decide the appropriate diameter and length of pigtail stent and its number.

As shown in Video 1, whenever feasible, we explored the cavity with the endoscope to clean and to perform necrosectomy (as reported by Lemmers et al.[4]) if necessary. All upper gastrointestinal exams were started with a standard gastroscope. A duodenoscope or colonoscope was used because of the larger operative channel if a $10 \mathrm{Fr}$ pigtail stent was needed. Meanwhile for lower gastrointestinal procedures, a standard colonoscope was always used. We believe the key to success for treatment of gastrointestinal fistulas is to accomplish complete internal drainage of any collection and to induce granulation tissue formation by reaction to pigtail, considered as a foreign body, in order to promote healing.

To simultaneously achieve both aforementioned goals, a Tandem ${ }^{\circledR}$ catheter (Boston Scientific, Mas- 
Table 1 Patient features and demographics.

\begin{tabular}{|c|c|c|c|c|c|c|}
\hline $\begin{array}{l}\text { Patient } \\
\text { number }\end{array}$ & Sex & Age & Pathology & Type of surgery & $\begin{array}{l}\text { Surgical/ } \\
\text { radiological drainage }\end{array}$ & Localization of fistula \\
\hline 1 & $\mathrm{~F}$ & 69 & Gallbladder tuberculosis & $\begin{array}{l}\text { Cholecystectomy with } \\
\text { resection of duodenal wall }\end{array}$ & Surgical & Duodenal bulb \\
\hline 2 & M & 65 & Gallstones and Crohn & Cholecystectomy & Surgical & Second duodenum \\
\hline 3 & $\mathrm{~F}$ & 58 & Gastric cancer & Total gastrectomy & N/A & Esophago-jejunal anastomosis \\
\hline 4 & M & 52 & Gastric cancer & Total gastrectomy & N/A & Esophago-jejunal anastomosis \\
\hline 5 & M & 78 & Rectal cancer & Total mesorectal excision & Surgical & Colorectal anastomosis \\
\hline 6 & $\mathrm{M}$ & 90 & Diverticulitis & Sigmoidectomy & N/A & Colorectal anastomosis \\
\hline 7 & $\mathrm{~F}$ & 67 & Gallstones & Cholecystectomy & Surgical & Duodenal bulb \\
\hline 8 & M & 75 & K cardia & Ivor-Lewis & Surgical & Esophago-gastric Anastomosis \\
\hline 9 & M & 67 & Gastric cancer & Total gastrectomy & N/A & Esophago-jejunal anastomosis \\
\hline 10 & M & 59 & Esophageal diverticula & Diverticulotomy & Surgical & Esophageal Staple line \\
\hline 11 & $\mathrm{~F}$ & 49 & Retroperitoneal cancer & Tumorectomy & Surgical/ radiological & Second duodenum \\
\hline
\end{tabular}

sachusetts, Boston, USA) was used to cannulate the defect and after guidewire insertion, one or more double pigtail stents (Advanix ${ }^{\circledR}$ Boston Scientific, Massachusetts-Boston-USA or Visio ${ }^{\circledR} \mathrm{G}$ flex Europe, Nivelles-Belgium) were deployed ( $\bullet$ Video 2 ). As a general rule, if an external drainage is present (surgical or radiological) the goal of EID is to reverse the drainage direction in order to remove early external drain, so a short stent usually is sufficient if no external drainage is present; one or two longer stents must be deployed to achieve complete cavity drainage.

In five of 11 patients a jejunal feeding tube was left in place. Meanwhile six patients were allowed an oral diet the day after the procedure. Systematic control was performed at 4 to 6 weeks in order to evaluate the evolution of the collection and to modify stent size according to the reduction of the cavity. A check endoscopy is important not only to evaluate treatment progression in order to adapt internal drainage, but also to promote healing by inducing trauma in the pseudocavity with the exchange of the double pigtail stents. After the first check endoscopy, oral diet resumption was allowed in four of five patients.

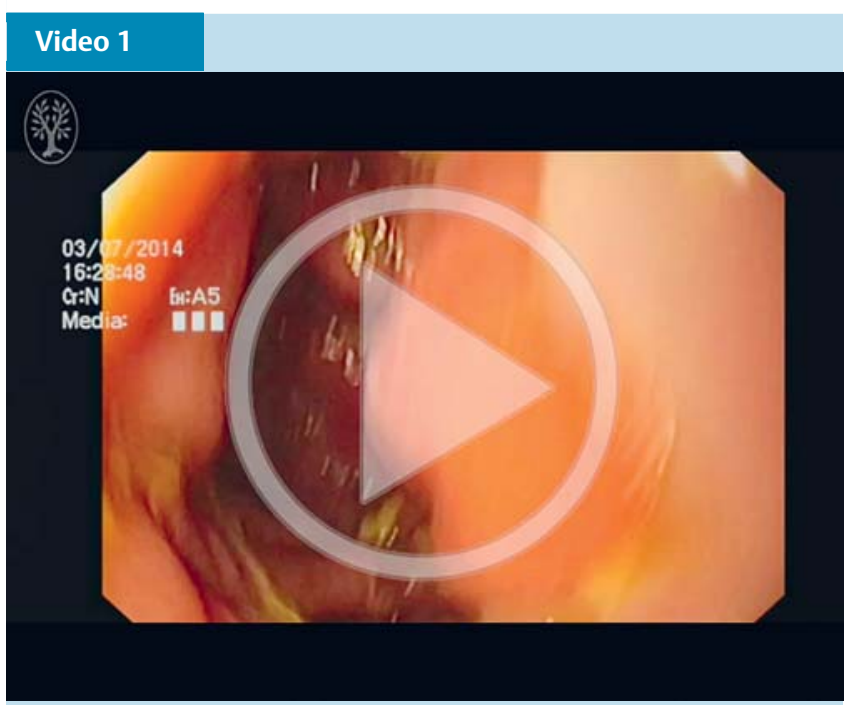

Endoscopic inspection of an esophago-jejunal anastomotic dehiscence of half the circumference with a concomitant mediastinal collection. Online content including video sequences viewable at: http://dx.doi.org/10.1055/s0042-105206

\section{Results}

$\nabla$

Technical success was defined by achievement of all the following steps: visualization of fistula orifice, opacification of fistula and collection, selective catheterization, and deployment of the double pigtail stent. Technical success was achieved in all patients (100\%). However, the day after the procedure, one patient presented with a perforation. This complication was not detected during the procedure and emergency surgery was required for esophago-jejunal anastomosis fistula. A redo of the anastomosis was successfully performed. Clinical success was defined as follows: no signs of sepsis after oral diet resumption, no extravasation of medium contrast at follow-up procedures (endoscopy, CT scan, barium enema) and absence of enteral liquid output from the external drainage/fistula tract.

Seven patients were found to be healed at first check endoscopy after an average of 35.6 days of treatment (range 28-58) ( $\bullet$ Fig. 1); one patient was healed at the second check (60 days) and the last patient was healed after three endoscopic sessions (90 days). We reported one clinical failure consisting of one patient still under treatment after 570 days. That patient under-

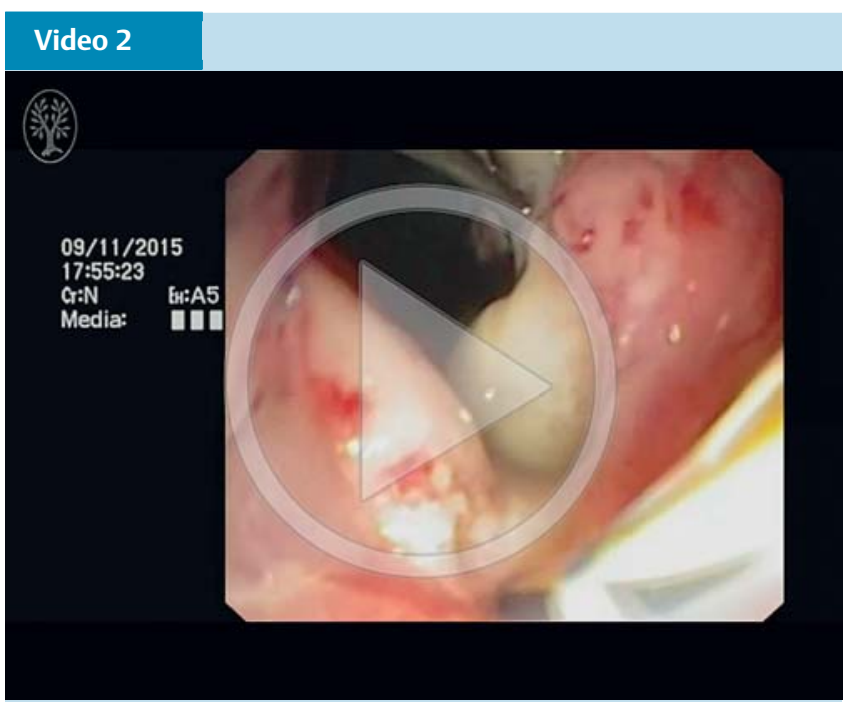

Endoscopic Internal drainage with deployment of two double pigtail stents to treat an anastomotic dehsicence following total gastrectomy. Online content including video sequences viewable at: http://dx.doi.org/10.1055/s0042-105206 


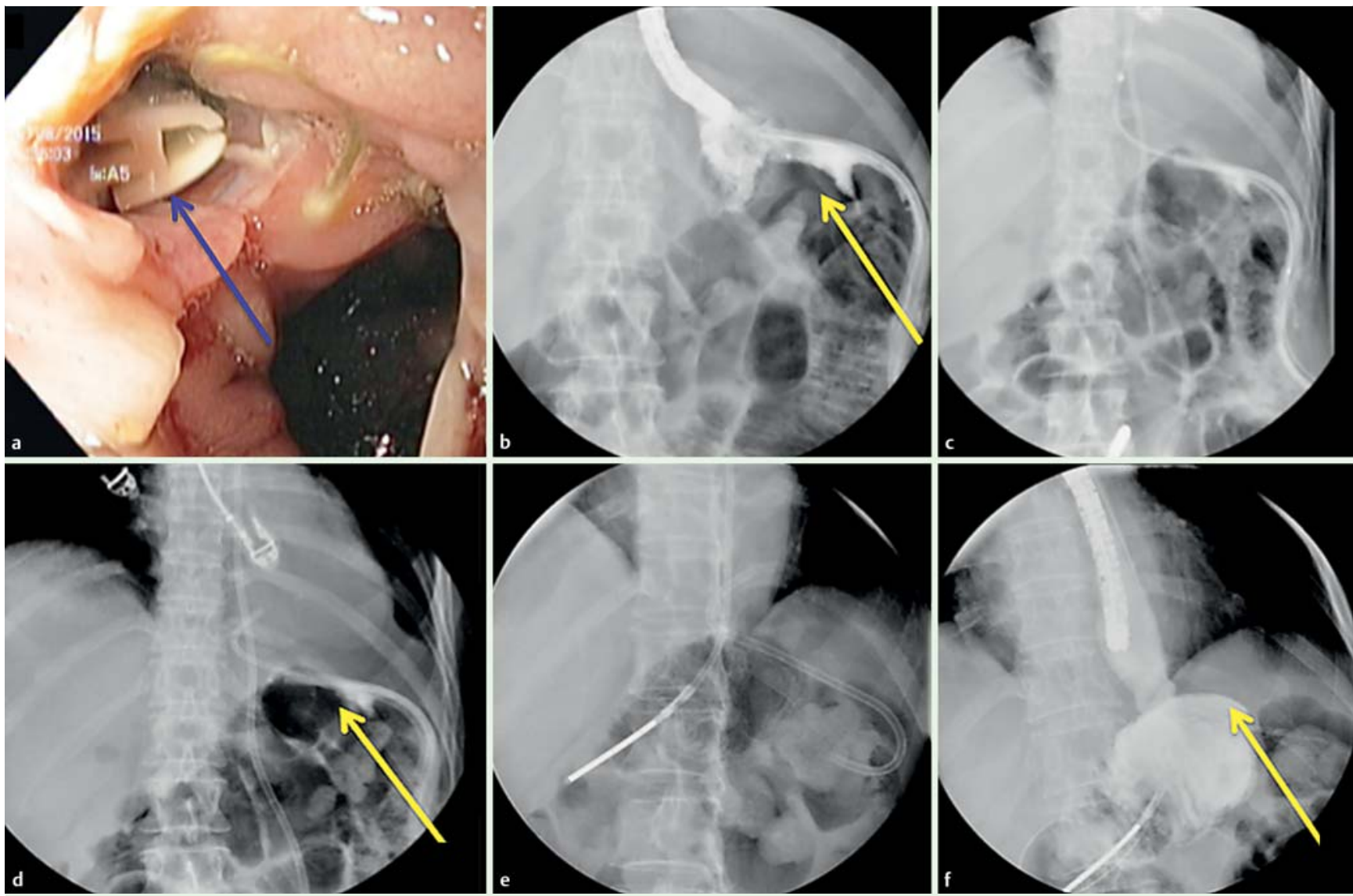

Fig. 1 a Endoscopic view of esophago-jejunal anastomotic fistula with surgical drainage in place (red arrow). b Radiological view of esophago-jejunal anastomotic fistula with surgical drainage in place (yellow arrow). c Deployment of double pigtail stent. $\mathbf{d}$ Double pigtail stent and feeding tube in place (yellow arrow). e Control after 28 days; removal of the surgical drainage. f Healed fistula with no extravasation of medium contrast (yellow arrow).

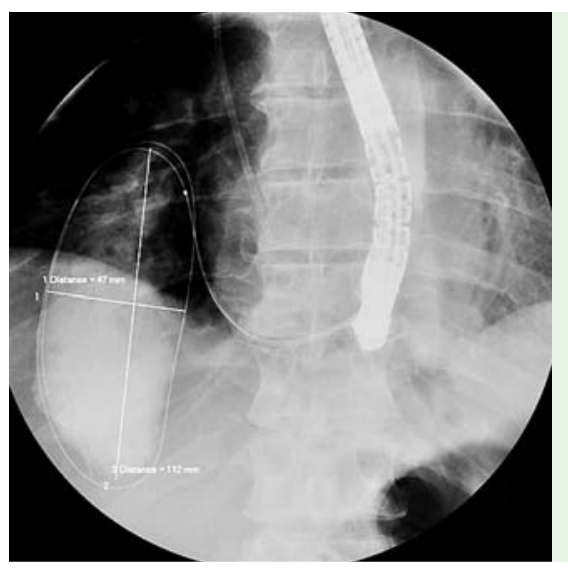

Fig. 2 Radiological visualization of perianastomotic collection.

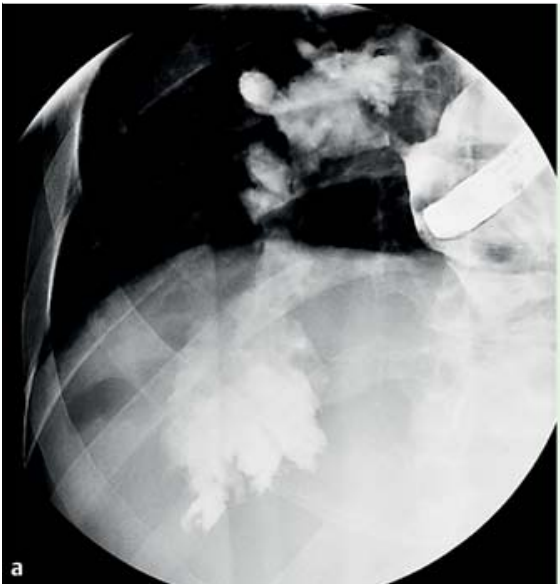

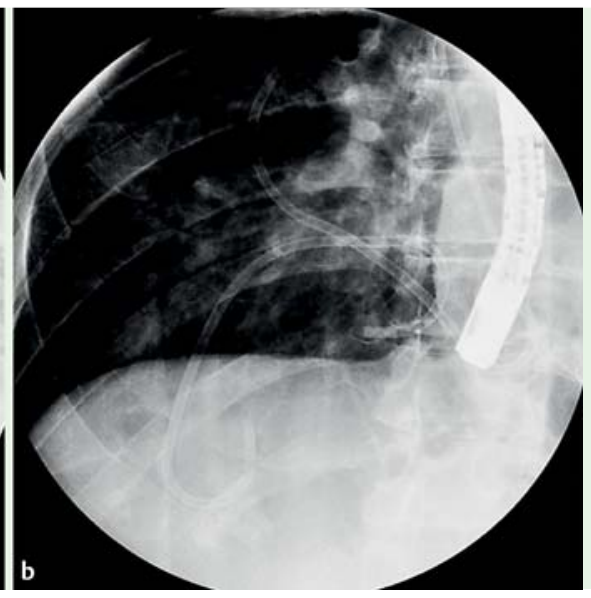

went total gastrectomy for T3N + gastric cancer and developed an anastomotic dehiscence of half of the circumference with a concomitant mediastinal collection after 11 days $(\bullet$ Video 1 and - Fig. 2). EID permitted delivery of two pigtail stents. After three endoscopic sessions and two stent replacements (90 days), there was no extravasation of contrast medium and the stents were removed. Unfortunately after 8 months the patient was readmitted to our hospital for septic shock. CT scan again showed a $10-\mathrm{cm}$ mediastinal collection due to an anastomotic fistula, and EID therefore was repeated ( $\bullet$ Fig.3). The span of time between endoscopic sessions was extended to 8 weeks instead of 4 weeks and he was allowed an oral diet.

Fig. 3 a Mediastinal collection measuring $10 \mathrm{~cm}$ due to an anastomotic fistula. b Endoscopic internal drainage treatment. 
Table 2 Type of scope used, number of pigtails deployed, overall and average endoscopy sessions, feeding method and outcome

\begin{tabular}{|clllll|}
$\begin{array}{l}\text { Patient } \\
\text { number }\end{array}$ & Endoscope & $\begin{array}{l}\text { Overall pig- } \\
\text { tail number }\end{array}$ & $\begin{array}{l}\text { Number of endo- } \\
\text { scopic sessions }\end{array}$ & Feeding & Outcome and days of treatment \\
\hline 1 & Duodenoscope & 4 & 4 & $28 \mathrm{~d}$ SNJ then Normal & P, 90 days \\
\hline 2 & Duodenoscope & 1 & 2 & Normal & P, 28 days \\
\hline 3 & Colonoscope & 1 & 2 & Normal & P, 28 days \\
\hline 4 & Duodenoscope & 28 & 14 & 60 days SNJ than Normal & N, UT, PIGTAIL in place (510 d) \\
\hline 5 & Colonoscope & 1 & 2 & Normal & P, 40 days \\
\hline 6 & Colonoscope & 2 & 2 & Normal & P, 36 days \\
\hline 7 & Duodenoscope & 1 & 2 & Normal & P, 58 days \\
\hline 8 & Gastroscope & 1 & 2 & SNJ & P, 28 days \\
\hline 10 & Colonoscope & 1 & 1 & SNJ & N, perforation on Day 1. Emergency surgery \\
\hline 11 & Colonoscope & 3 & 3 & SNJ 28 days than normal & P, 60 days \\
\hline
\end{tabular}

P: positive; N: negative; UT: under treatment.
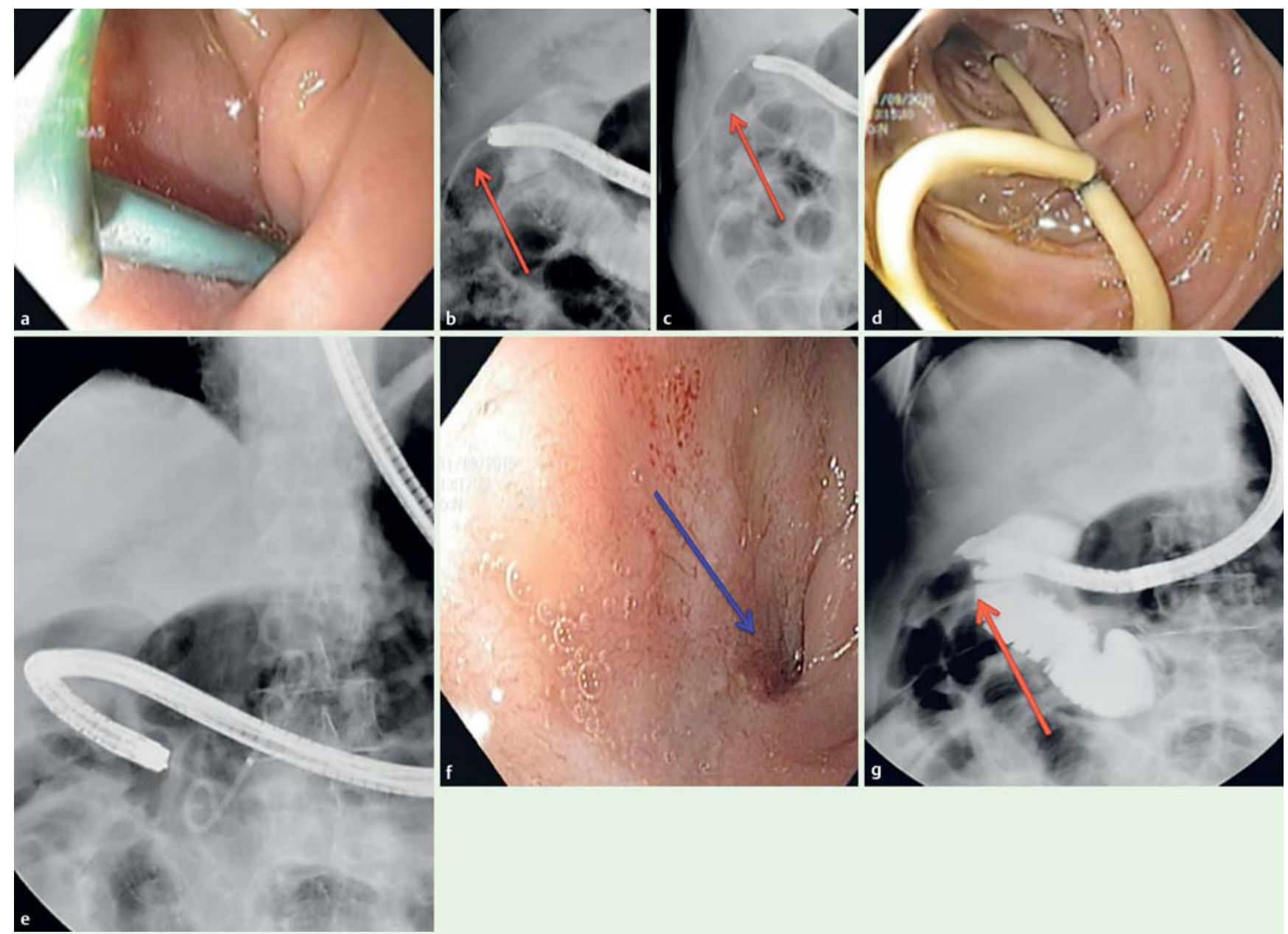

Fig. 4 a Endoscopic view of double pigtail stent deployed in the duodenal bulb for duodenal fistula. b Persistence of chronic fistula (red arrow). c Deployment of a double pigtail stent (red arrow). $\mathbf{d}$ Endoscopic view of a double pigtail stent migrated in the duodenal lumen 28 days after EID treatment. e Radiological view of double pigtail stent migration in the duodenal lumen. $\mathbf{f}$ Endoscopic view of healed orifice fistula (blue arrow). $\mathbf{g}$ Radiological control of healed orifice fistula (red arrow).

- Table 2 lists the type of scope used, the number of pigtail stents deployed, the number of overall and average endoscopy sessions, feeding method, and outcomes

If present, the external drainage tube was removed before the first check endoscopy (4-6 weeks after start of EID). Overall we achieved clinical success in nine patients after an average of 44 days (28-90) treatment with a median of 2.3 endoscopic sessions $(2-4)$ and deployment of 1.8 pigtail stents per patient
(1-4). The overall clinical success rate was $82 \%$, with EID failure in two of 11 patients (18\%). No stent-related bleeding or stent migrations inside the abdominal cavity were observed in the study. At a median follow up of 291 days (10-970) all patients are on an oral diet and symptom-free. 


\section{Discussion}

\section{$\nabla$}

Leak and fistula following gastrointestinal surgery are usually treated minimally invasively with drainage tubes, oral alimentary stream diversion, and/or plugging. Several endoscopic treatments have been proposed to achieve this aim directly or indirectly. The most common approaches are insertion of temporary covered self-expandable metal stents (cSEMS); clipping; plugging; gluing; and vacuum therapy $[5,6,7]$. Overall clinical success rate varied greatly among these different techniques without assurance of optimal results. Moreover, the rate of success decreases with the interval between initial surgery and endoscopic treatment.

The most common treatment is with deployment of cSEMS, but they are burdened by a high rate of migration due to altered anatomy and absence of stenosis coupled with the physiologically large diameter of the gastrointestinal tract. These factors do not allow the cSEMS to guarantee watertight exclusion of a leak. Even if the stent remains in place, fluids pass in the space between the external sheets of the stent and the wall of the gastrointestinal lumen.

Endoscopic Internal drainage of intraabdominal collection was first used in the $1980 \mathrm{~s}$ by biliopancreatic endoscopists to treat pancreatic pseudocyst. One or several pigtails were introduced under endoscopic vision, puncturing the bulging collection in order to perform an anastomosis between the cavity and the gastrointestinal tract. Later, thanks to the advent of interventional endoscopic ultrasound, we had the opportunity to drain and create anastomosis between an abdominal collection that was neither bulging nor endoscopically visible and the gastrointestinal tract.

Today newly designed, lumen apposing, fully covered self-expandable metal stent (LA-FCSEMS) may be used for this purpose [8], enabling the emptying of the collection with resolution of this virtual third space. The EID procedure is based on the same concept even if the access - the wall defect (fistula-leak orifice) is already present. In our previous studies we demonstrated that a pigtail stent acts as a foreign body on the edge of the leak and inside the cavity, promoting its reepithelialization $[1,9,10]$. EID is a direct way of treating fistula.

The new concept in our series is that EID can be used to internally drain perianastomotic fluid collection all along the gastrointestinal tract (esophagus, duodenum, colon). Application of pigtail drainage is feasible even in the colon and rectum, as shown in an interesting article by Calzolari et al. [11]. We decided to systematically evaluate each patient after 4 to 6 weeks because pigtail stents have a small caliber and can easily get blocked. More importantly we believe that the stent being a foreign body promotes granulation tissue formation, as demonstrated by spontaneous double pigtail migration ( Fig.4).
It is well known that early management of fistula should always be advocated because it correlates with higher clinical success rates. Therefore, we advocate EID as an early first-line treatment. Although EID often requires multiple endoscopic sessions, it is cost-saving compared to SEMS.SEMS are very costly and require multiple endoscopic sessions. Based on our experience, EID should be considered as an effective first option for leaks throughout the gastrointestinal tract.

\section{Conclusions \\ $\nabla$}

EID is a valuable and efficient technique not only for leaks following bariatric surgery but even for leaks and fistulas following other type of upper and lower gastrointestinal surgery. As long as EID can achieve access to the fluid collection, internal drainage can be achieved all along the gastrointestinal tract.

Competing interests: Dr. Donatelli is a consultant for Boston Scientific.

\section{References}

1 Donatelli G, Dumont JL, Cereatti $F$ et al. Treatment of leaks following sleeve gastrectomy by endoscopic internal drainage (EID). Obes Surg 2015; 25: $1293-1301$

2 Pequignot A, Fuks D, Verhaeghe $P$ et al. Is there a place for pigtail drains in the management of gastric leaks after laparoscopic sleeve gastrectomy? Obes Surg 2012; 22: $712-720$

3 Rogalski P, Daniluk J, Baniukiewicz A et al. Endoscopic management of gastrointestinal perforations, leaks and fistulas. World J Gastroenterol 2015; 21: $10542-10552$

4 Lemmers A, Tan DM, Ibrahim $M$ et al. Transluminal or percutaneous endoscopic drainage and debridement of abscesses after bariatric surgery: a case series. Obes Surg 2015; 25: 2190-2219

5 Swinnen J, Eisendrath P, Rigaux J et al. Self-expandable metal stents for the treatment of benign upper GI leaks and perforations. Gastrointest Endosc 2011; 73: 890-899

6 Toussaint E, Eisendrath P, Kwan V et al. Endoscopic treatment of postoperative enterocutaneous fistulas after bariatric surgery with the use of a fistula plug: report of five cases. Endoscopy 2009; 41: 560563

7 Keren D, Eyal O, Sroka $G$ et al. Over-the-scope (OTSC) system for sleeve gastrectomy leaks. Obes Surg 2015; 25: $1358-1363$

8 Alcaide N, Vargas-Garcia AL, de la Serna-Higuera C et al. EUS-guided drainage of liver abscess by using a lumen-apposing metal stent (with video). Gastrointest Endosc 2013; 78: 941 - 942

9 Donatelli G, Catheline JM, Dumont JL et al. Outcome of leaks following sleeve gastrectomy based on a new alghoritm addressing leak size and gastric stenosis. Obes Surg 2015; 25: $1258-1260$

10 Donatelli G, Ferretti S, Vergeau BM et al. Endoscopic internal drainage with enteral nutrition (EDEN) for treatment of Leaks following sleeve gastrectomy. Obes Surg 2014; 24: 1400-1407

11 Calzolari C, Barret M, Rahmi G et al. Diverticular abscess successfully treated by endoscopic transluminal drainage. Clin Res Hepatol Gastroenterol 2014; 38: e17-e18 